LA W RENCE LIVERMORE N A TIONAL LABORATORY

Determining fission cross

sections of short lived nuclei in the actinide region

J. T. Burke, W. Younes, I. J. Thompson

November 22, 2011 
This document was prepared as an account of work sponsored by an agency of the United States government. Neither the United States government nor Lawrence Livermore National Security, LLC, nor any of their employees makes any warranty, expressed or implied, or assumes any legal liability or responsibility for the accuracy, completeness, or usefulness of any information, apparatus, product, or process disclosed, or represents that its use would not infringe privately owned rights. Reference herein to any specific commercial product, process, or service by trade name, trademark, manufacturer, or otherwise does not necessarily constitute or imply its endorsement, recommendation, or favoring by the United States government or Lawrence Livermore National Security, LLC. The views and opinions of authors expressed herein do not necessarily state or reflect those of the United States government or Lawrence Livermore National Security, LLC, and shall not be used for advertising or product endorsement purposes.

This work performed under the auspices of the U.S. Department of Energy by Lawrence Livermore National Laboratory under Contract DE-AC52-07NA27344. 


\title{
Determining fission cross sections of short lived nuclei in the actinide region
}

\author{
J.T. Burke, W. Younes and I.J. Thompson \\ Technical Report \\ November 14, 2011 \\ IM 539900
}

\section{Executive Summary}

This brief report summarizes the experimental and nuclear theory and modeling approach needed to determine cross sections on short-lived nuclei. Previous work on actinide $(\mathrm{n}, \mathrm{f})$ cross sections has shown the technique to be accurate to $20 \%$ below $E_{n}=1 \mathrm{MeV}$, and $10 \%$ above. Measurements are proposed that will meet and exceed this level of accuracy for $(\mathrm{n}, \mathrm{f})$ cross sections on ground and excited states by constraining critical model uncertainties.

\section{Introduction}

Neutron induced cross sections on short lived actinides can be difficult if not impossible to measure depending on the half-life of the target nucleus. In these cases direct reaction data on stable targets coupled with nuclear theory and modeling can be used to extract energy dependent fission cross sections. Over the past decade multiple avenues of research have proven this to be the case in the actinide region. Currently, fission cross sections determined this way have documented uncertainties of $20 \%<1 \mathrm{MeV}$ and $10 \%>1 \mathrm{MeV}$ [1].

Determination of cross-sections for short lived nuclei require a combined approach using both theory and experimental data. Theory must be able to predict the direct reaction population probability of a given spin and parity in the compound nucleus at a given energy, and must provide a reasonable fission model. Experiment must be able to measure an energy dependent probability for the channel being considered, for this report we are only considering the fission channel.

In the following sections we examine the theory \& modeling needs and then the experimental requirements.

\section{Theory and Modelling}

Research by Younes and Britt [1-2] has demonstrated that theory and modeling can be used to predict actinide fission cross sections from direct-reaction data. For example, for a (d,pf) surrogate reaction, the measured fission probability is given by

$$
P_{(d, p f)}\left(E_{x}\right)=\sum_{J^{\pi}} P_{(d, p)}\left(J^{\pi}\right) \times P_{f}\left(E_{x}, J^{\pi}\right)
$$

where the sum includes all spins $J$ and parities $\pi$ populated in the compound nucleus by the $(d, p)$ reaction. The population probability for each $\mathrm{J}$ is represented by $\mathrm{P}_{(\mathrm{d}, \mathrm{p})}\left(\mathrm{J}^{\pi}\right) \cdot \mathrm{P}_{\mathrm{f}}\left(\mathrm{E}_{\mathrm{x}}, J^{\pi}\right)$ is the probability that the compound nucleus with excitation 
energy Ex and spin-parity $J^{\pi}$ will fission. Direct reaction theory must predict $\mathrm{P}_{(\mathrm{d}, \mathrm{p})}\left(\mathrm{J}^{\pi}\right)$. The fission probability $\mathrm{P}_{\mathrm{f}}\left(\mathrm{E}_{\mathrm{x}} \mathrm{J}^{\pi}\right)$ is determined by adjustment of a fission model described below.

Once $\operatorname{P}_{f}\left(E_{x} J^{\pi}\right)$ has been determined it can be used to calculate the $(n, f)$ cross section of interest by the relation

$$
\sigma_{(n, f)}\left(E_{n}\right)=\sum_{J^{\pi}} \sigma_{C N}\left(E_{n}, J^{\pi}\right) \times P_{f}\left(E_{x}, J^{\pi}\right)
$$

where $\sigma_{\mathrm{CN}}\left(E_{n}, J^{T}\right)$ is the compound nucleus formation cross section for the neutron induced reaction and $E_{n}$ is the incident neutron energy.

The direct reaction population for a given spin and parity can be extracted from measurements of transfer reactions with target isotopes that are either stable or sufficiently long-lived. Depending on the desired compound nucleus, transfer reactions such as $(d, p)$ stripping or $(p, t)$ pickup may be suitable for such experiments. The $(d, p)$ stripping reaction can be accurately modeled theoretically. Since now, however, we need not just stripping to bound states but to unbound states, we need further theory to describe the competition between the escape (breakup) and the fusion (compound nucleus production) of the neutron in continuum states. Recent LLNL work by I. Thompson has developed the theory for partial fusion, and would be useful here. The $(p, t)$ pickup reaction is simpler in that it does not directly populate neutron continuum states, but models to predict the resulting $\left.P_{(\mathrm{p}, \mathrm{t})} U^{t}\right)$ probabilities does depend on the strength of neutron-neutron pairing in the target nucleus. For that purpose some account of neutron pairing needs to be evaluated as a parallel theory project. The best candidate for evaluating the two-neutron response function could well be the Auxiliary-Field Monte Carlo method developed in part by E. Ormand at LLNL. Otherwise we should try to apply Hartree-Fock-Bogoliubov mean-field pairing models or (better) projected shell models.

The $\mathrm{P}_{\mathrm{f}}\left(\mathrm{E}_{\mathrm{x}}, \mathrm{J}^{\pi}\right)$ terms are calculated using a double humped fission barrier which assumes statistical competition between the various channels (n-emission, gamma decay and fission) [2]. Information that would help improve the validity of this model includes measurements of the fission fragment anisotropy, transition states below and above the fission barrier. The approach outlined above has already been benchmarked against 9 measured cross sections with good agreement as can be seen in Figure 1. 


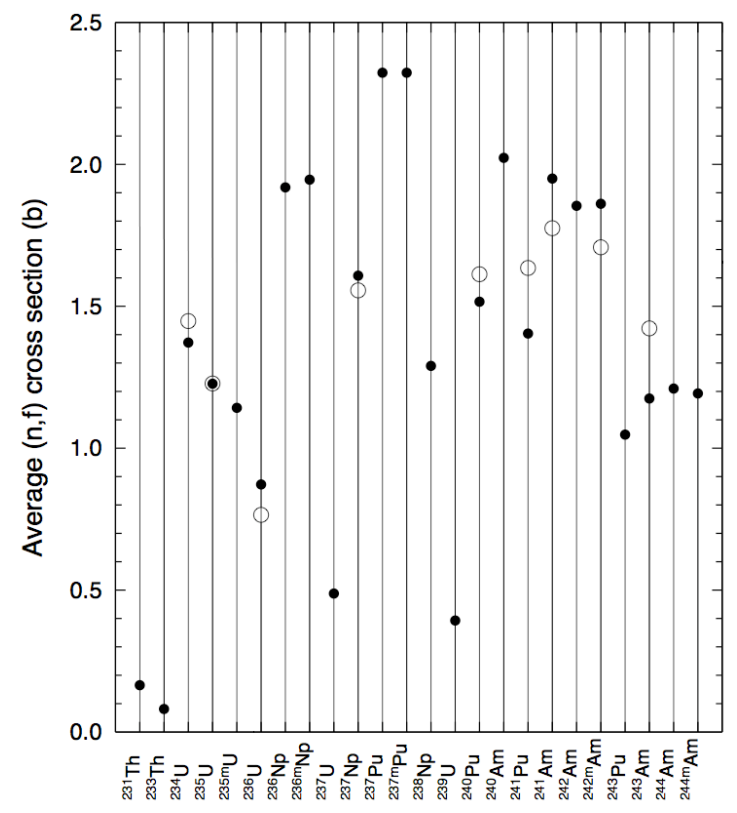

Figure 1. Summary of deduced $(\mathrm{n}, \mathrm{f})$ cross sections from Younes [1]. The points represent the cross section averaged over $1 \mathrm{MeV}$ up to $6 \mathrm{MeV}$. Solid circles represent the cross sections using this technique. The open circles represent cross sections from ENDF/B-VI.

The technique also determines the energy, spin and parity dependent fission probabilities that can be used to determine the cross section of excited states as can be seen in Figure 2. This was done explicitly for ${ }^{235 \mathrm{~m} U}$ by Younes and Britt [2]. The theoretical model was also put through a sensitivity study to identify the weaknesses of the method. The test are identified in Table I below and discussed in detail in the appendix of Younes and Britt [2]. The tests conclude that the cross section uncertainty is on the order of $\pm 20 \%$ for physically reasonable parameter values used in the determination of the $235 \mathrm{~m} U$ cross section. 


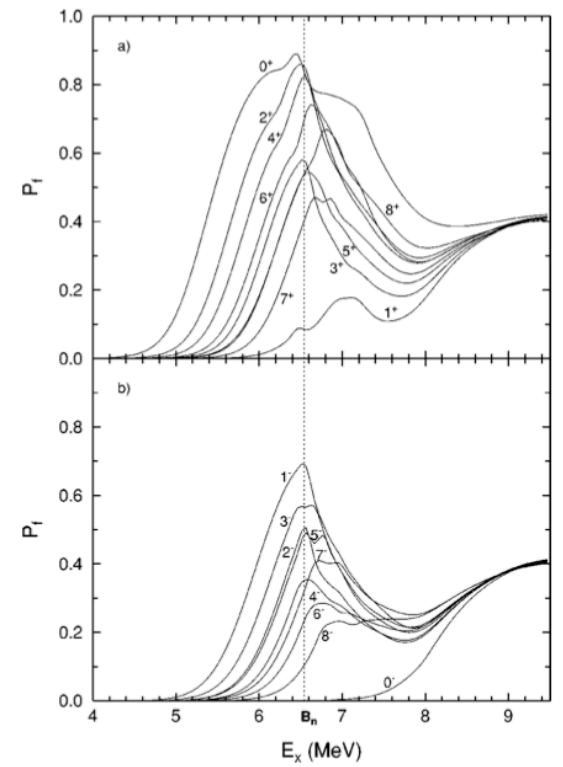

Figure 2. Fission probabilities for ${ }^{235} \mathrm{U}+\mathrm{n}$ determined from theory and direct reactions data. The positive-parity probabilities are shown in panel a) while the negative-parity probabilities are shown in panel b). Figure from Younes and Britt [2].

\begin{tabular}{|c|c|}
\hline Test No. & Description \\
\hline 1 & $\begin{array}{l}\text { Replaced discrete levels with continuum level density in } \\
{ }^{235} \mathrm{U} \text {. }\end{array}$ \\
\hline 2 & $\begin{array}{l}\text { Eliminated all two-phonon transition states above the } \\
\text { barriers. }\end{array}$ \\
\hline 3 & $\begin{array}{l}\text { Used transmission coefficients from the ABACUS } \\
\text { calculation. }\end{array}$ \\
\hline 4 & $\begin{array}{l}\text { Assumed twice as many positive- as negative-parity states } \\
\text { in } 1^{s t} \text { wells of }{ }^{236} \mathrm{U} \text { and }{ }^{235} \mathrm{U} \text {, affecting } \gamma \text { and neutron } \\
\text { decays as well as the formation processes. }\end{array}$ \\
\hline 5 & $\begin{array}{l}\text { Used constant } P_{(t, p)}\left(J^{\pi}\right) \text { population probability up to } J \\
=10 \text {, zero for } J>10 \text {. }\end{array}$ \\
\hline 6 & $\begin{array}{l}\text { Fit entire } E_{x} \text { energy range of } P_{(t, p f)} \text { data, varying all three } \\
\text { barrier heights. }\end{array}$ \\
\hline 7 & $\begin{array}{l}\text { Scaled } 1^{s t} \text {-well continuum level density by } 2.5 \text { in }{ }^{236} \mathrm{U} \text { and } \\
\text { by } 2.0 \text { in }{ }^{235} \mathrm{U} \text { to match known level spacings at the neutron } \\
\text { binding energy. }\end{array}$ \\
\hline 8 & $\begin{array}{l}\text { Used double-Lorentzian dependence for } \gamma \text {-ray strength } \\
\text { function instead of } E_{\gamma}^{3} \text { form in Eq. (10). }\end{array}$ \\
\hline 9 & $\begin{array}{l}\text { Raised all two-phonon energies by } 30 \% \text { for transition states } \\
\text { above the barriers. }\end{array}$ \\
\hline 10 & $\begin{array}{l}\text { Scaled the discrete-level contribution to } N_{n} \text { by the factor } \\
0.58 \text { to eliminate the dip in the calculated } P_{(t, p f)}\left(E_{x}\right) \text { curve. }\end{array}$ \\
\hline 11 & Set $\hbar \omega_{A}=0.65$. \\
\hline 12 & Set $\hbar \omega_{B}=0.90$. \\
\hline 13 & $\begin{array}{l}\text { Turned off width-fluctuation correction for both fission and } \\
\text { neutron emission. }\end{array}$ \\
\hline 14 & Fit without $B_{I I}$ barrier. \\
\hline
\end{tabular}

Table I. Model dependent sensitivity test suite used in Younes and Britt [2] 


\section{Experiment}

Direct reaction experiments require charged particle beams and silicon detectors to measure the energy and angle of the scattered particles. The energy of the scattered particles tell us the energy we have imparted into the nucleus. The angle of the scattered particle allows us to reconstruct the kinematics of the reaction. Combined together we can vary look at different energies and angles to examine the excitation and spin dependent physics of a given nucleus.

The data used for the above studies was actually obtained in the 1960's and 1970's from the Los Alamos Van de Graaff accelerator. The detector setup used for the measurements by Britt, Rickey and Hall [3] is shown in Figure 3. The apparatus consisted of a single silicon telescope used to detect the energy, angle and identify the scattered particle type $(\mathrm{p}, \mathrm{d}, \mathrm{t})$. The fission fragments were detected by a series of 8 fission detectors surrounding the target. In a later paper by Cramer and Britt, the silicon telescope was mounted on a rotatable arm allowing multiple particle angles to be measured.

Improvements in precision manufacturing and cost reductions have allowed high purity silicon detectors to manufactured in ever increasing thicknesses and dimensions. Currently the HYDRA array, Figure 4, chamber makes use of 14 silicon telescopes for particle measurement. The telescope actually consists of two Ortec detectors; $\mathrm{dE}$ is 200 micron thick a pure silicon and the E detector is a 5000 micron thick lithium drifted silicon detector (SiLi). This now enables experimenters to fully stop and record the energy of protons up to $30 \mathrm{MeV}$ in energy. The benefit of this technology is that we can measure a higher excitation energy of nuclei than was previously accessible. The HYDRA array allows a simultaneous measurement of 14 different scattering angles and excitation energies up to $15 \mathrm{MeV}$ for proton like scattered particles and up to $30 \mathrm{MeV}$ excitation energies for ${ }^{3} \mathrm{He}$ and ${ }^{4} \mathrm{He}$ particles. The principle role of the telescope array is to measure the individual scattered particles energy and angle. These events are used to determine the energy and spin dependent population of the excited nuclei under investigation. They are also used to validate the theoretical prediction of the direct reaction cross section (ie: differential scattering cross section). 


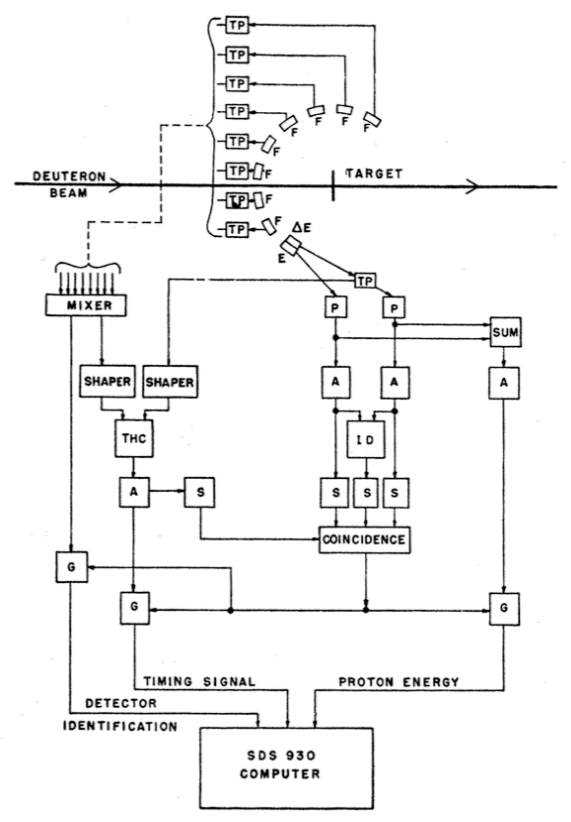

Figure 3. Apparatus used by Britt [3] to measure the transition-state spectra, angular correlations and fission probabilities for $\mathrm{U}$ and $\mathrm{Pu}$.

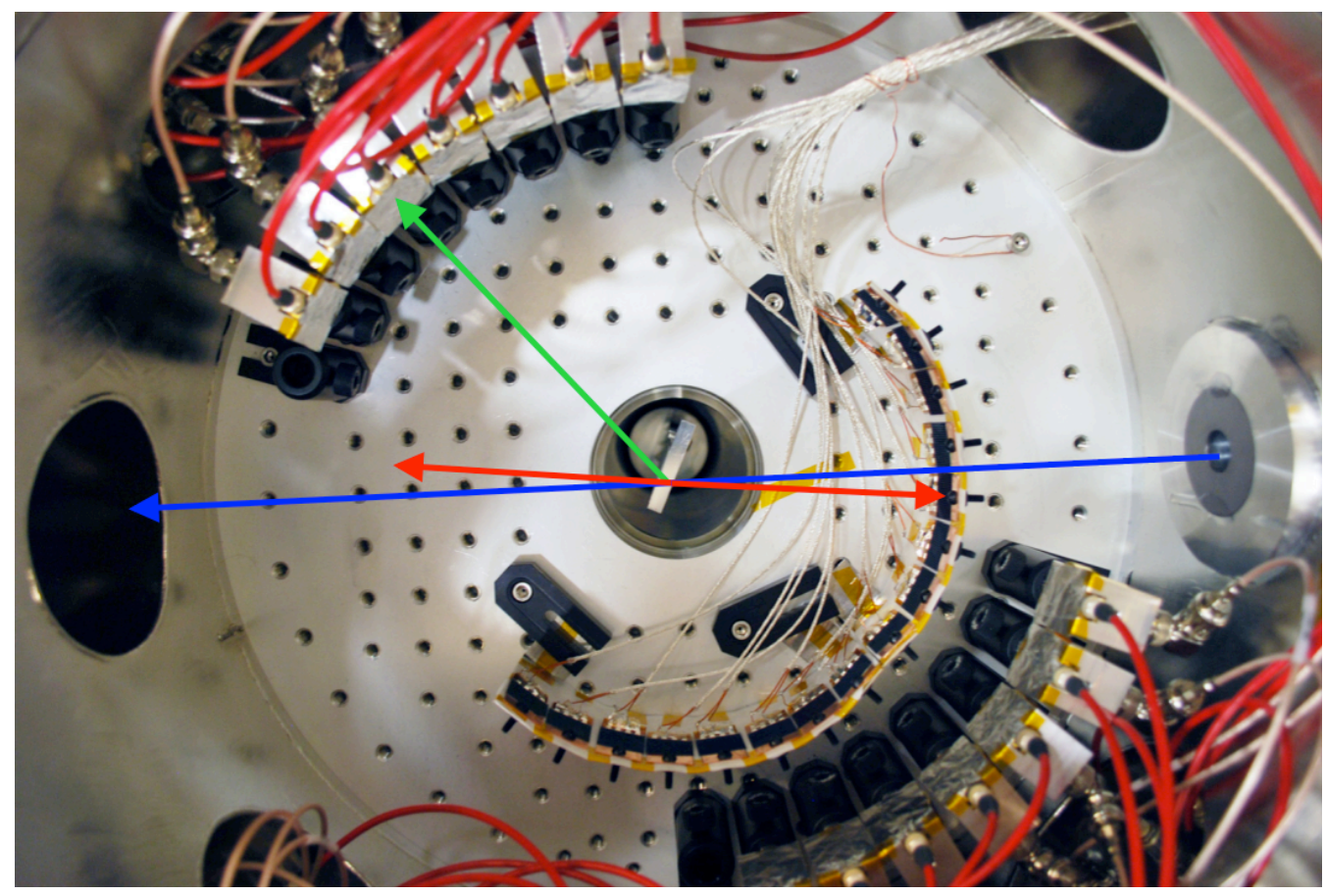

Figure 4. HYDRA chamber and apparatus currently consists of $14 \mathrm{dE}-\mathrm{E}$ silicon telescopes covering $30^{\circ}$ to $160^{\circ}$. An inner semi-circle of mono-crystalline solar cells can be seen in the bottom right quadrant. The blue arrow shows the primary beam 
path, the green arrow shows the scattered particle trajectory to the detector, the red back-to-back arrows show the fission fragment trajectories. Setup used as a test run and commissioning of our new digital data acquisition system.

The HYDRA array simultaneously measures the fission fragments using an array of mono-crystalline solar cells arranged in a semi-circle around the target. A total of 600 mono-crystalline uncoated solar cells are available for experiments. Currently the array consists of 16 fission detectors located in the same plane as the silicon telescopes. This is the ideal way to measure the fission fragment angular distribution, but not sufficient to measure the total fission probability. In order to complete the fission array, a fraction of the remaining solar cells must be instrumented and installed out of the silicon telescope detector plane. This will insure that the total fission probability as well as the fission fragment distribution can be simultaneously measured. Both elements are key to the understanding of the fission model used to determine the direct reaction fission cross sections.

A test run of ${ }^{239} \mathrm{Pu}(\mathrm{d}, \mathrm{p})$ was used in August 2011 as a commissioning run of our new digital data acquisition (DAQ) system and the HYDRA array. The digital acquisition modules (VF48s) did not perform as we expected them to. The DAQ system has been redesigned and we have reverted to modern VME based analogue ADCs which we anticipate will be complete in December 2011. The DAQ is common to all experimental efforts for direct reaction research at LLNL. Robert Casperson (PD) was able to salvage the data from the test run and it shows similar results to Britt et al with only 12 hours of run time, see Figure 5.

In 12 hours we were able to accumulate 500 counts ( $4.5 \%$ stat. unc.) per 200 $\mathrm{keV}$ bin right at the neutron separation energy. Sn is the equivalent energy of a "zero" energy neutron capture. The number of detectors in the fission array should be increased by a factor of 8 to 128 channels. The energy bins need to be subdivided to $100 \mathrm{keV}$ per channel which results in an overall factor of 4 increase in the data per $100 \mathrm{keV}$ bin. Furthermore, we can run the experiment for 10 days instead of 12 hours and gain a factor of 20 . This would result in statistics on the order of ( $80 \mathrm{x} 250$ counts $/ 100 \mathrm{keV}=20000$ counts $/ 100 \mathrm{keV})$ or a statistical uncertainty of $<1 \%$. The total number of counts needs to be further divided by the 14 silicon telescopes. Assuming it is a linear distribution, we still can obtain (20000/14 1400 counts $/ 100 \mathrm{keV}$ bin) statistics on the order of $2.7 \%$. Clearly systematic effects of the measurement will come into play larger or comparable to the statistical uncertainties. In particular, calibration of the efficiency of the fission fragment detectors is a key uncertainty. However commercial calibrated sources such as ${ }^{252} \mathrm{Cf}$ are available with NIST traceable activity and uncertainties on the order of $1 \%$ for 1 sigma. 

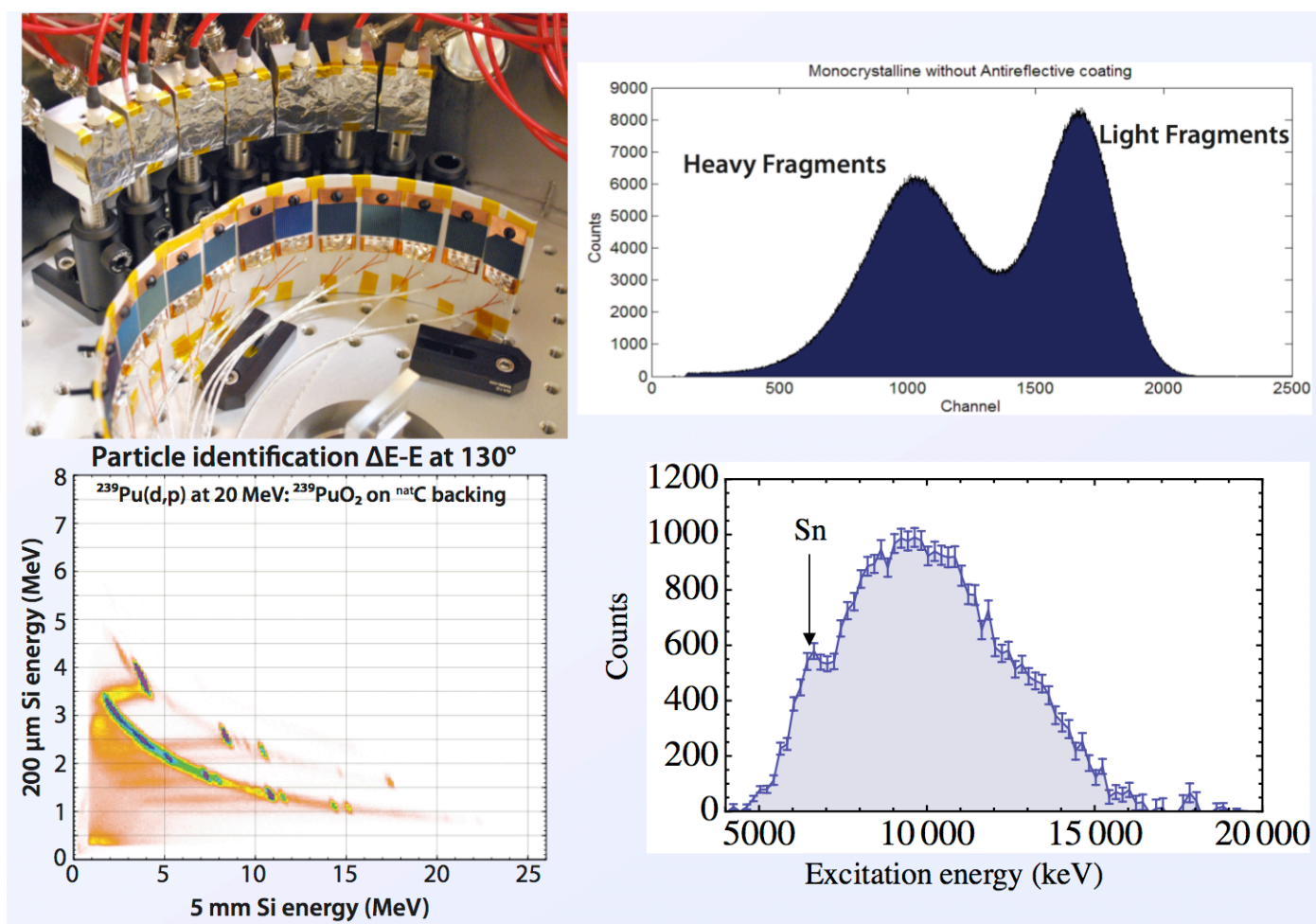

Figure 5. Test run data for $20 \mathrm{MeV}$ deuterons incident on a ${ }^{239} \mathrm{Pu}$ target. Top left shows the HYDRA array with fission detectors. Top right shows the fission spectrum obtained with a ${ }^{248} \mathrm{Cm}$ source for the fission detectors. Bottom left is the particle identification plot from a single telescope (not gated on fission). Bottom right shows a proton-fission gated coincidence spectrum for all the detectors in the HYDRA array for a 12 hour test run.

The mono-energetic highly collimated charged particle beams needed for this type of research are available at the Texas A\&M University Cyclotron Institute located in College Station Texas, see Figure 6. A Memorandum of Understanding is under final review for a collaboration between Texas A\&M and LLNL. The MOU allows the experimental nuclear physics group at LLNL to perform experiments at TAMU-CI. In exchange the TAMU-CI users are allowed to use the apparatus for locally run experiments. The MDM spectrometer has a precision scattering chamber almost identical to the current HYDRA array chamber. It has a beam focal point of 1 $\mathrm{mm}$ diameter and beam energy uncertainty of $\sim 30 \mathrm{keV}$. These beam conditions already exist and were necessary to enable proper functioning of the MDM spectrometer conditions appropriate. Upstream of the MDM spectrometer there is a Beam Analysis System to properly reduce the energy spread of the beam from the K500 Cyclotron. This is followed by sets of collimators and active focusing elements consisting of magnetic quadrupole and vertical \& horizontal steering magnets. 


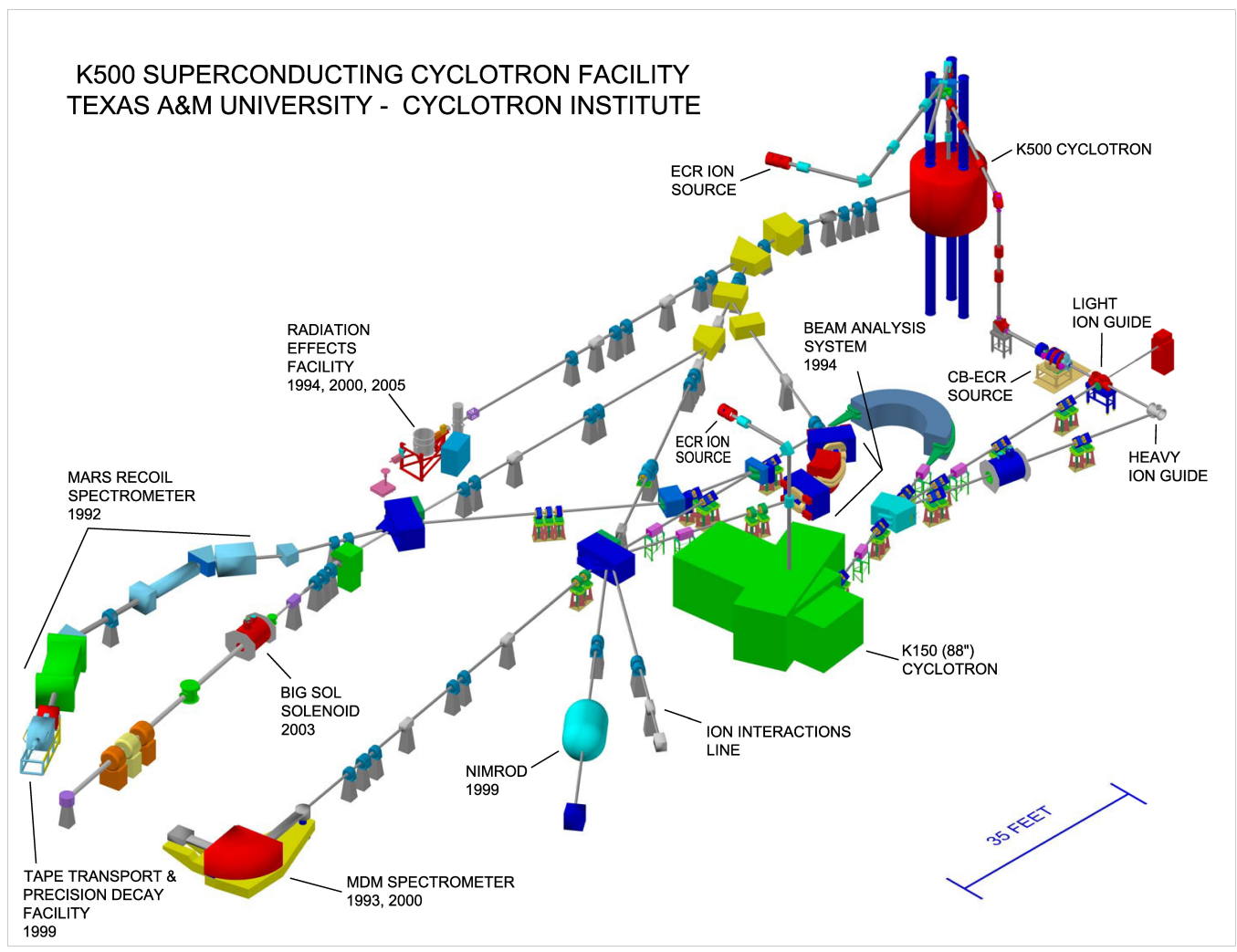

Figure 6. Schematic layout of the Texas A\&M Cyclotron Institute's cyclotrons and beamlines. The STARS/Liberace array will be located on the Ion Interactions Line. The HYDRA array can be located in the MDM spectrometer scattering chamber.

\section{Research Plan}

We propose to measure the ground state and excited state fission cross section of ${ }^{239} \mathrm{Pu}+\mathrm{n}$ using up to three different reactions in the following order; ${ }^{239} \mathrm{Pu}(\mathrm{d}, \mathrm{p}),{ }^{242} \mathrm{Pu}(\mathrm{p}, \mathrm{t})$ and ${ }^{240} \mathrm{Pu}\left(\mathrm{p}, \mathrm{p}^{\prime}\right)$. Theory and modeling will have to be developed concurrently to predict the population probabilities. We will cross check the fission theory model by measuring fission fragment angular distributions below and above the neutron separation energy. We will determine the energy dependent level density parameters.

Due to the needed time to develop theory and field \& analyze the data from the experiments, this program will take approximately 3 years to fully complete. At each stage of experimental results and/or theory developments, results and progress will be reported to the sponsor. 


\section{Summary}

In summary, the theoretical groundwork has been determined for deducing cross sections from short-lived nuclei. More theoretical work is needed to expand on the present theory and modeling capabilities to be able to include the nuclear reactions needed for this study. The experimental apparatus exists and is adequate for the proposed research following completion of the fission array. Our goal is to examine multiple entrance channels to constrain the final cross section to better than $20 \%$ uncertainty below $1 \mathrm{MeV}$ and better than $10 \%$ above $1 \mathrm{MeV}$ as has already been demonstrated. We will use the ground state cross section that has been well measured to validate/benchmark the approach.

\section{Acknowledgements}

This work performed under the auspices of the U.S. Department of Energy by Lawrence Livermore National Laboratory under Contract DE-AC52-07NA27344.

\section{References}

1. W. Younes and H.C. Britt, "Simulated Cross Sections on Various Th, U, Np, Pu and Am Targets", International Conference on Nuclear Data for Science and Technology, AIP (2005)

2. W. Younes and H.C. Britt, "Neutron-induced fission cross sections simulated from (t,pf) results", Phys. Rev. C 67, 024610 (2003)

3. H.C. Britt, F.A. Rickey, Jr., and W.S. Hall, "Characteristics of the Transition-State Spectra for 234U, 236U, and 240Pu from (d,pf) and (t,pf) Angular-Correlation Experiments", Phys. Rev. 175, 1525 (1968) 